\title{
Employment Status of Patients with ESRD and the Subsequent treatment modalities in the United States
}

\author{
John D Sullivan* \\ Boston University, Metropolitan College, United States \\ *Corresponding author: John D Sullivan, Boston University, Metropolitan College, United States
}

\section{Introduction}

End Stage Renal Disease impacts the lives of over 725,000 Americans and their families. In 1972, despite the cost of supporting a rapidly growing chronic condition at the time, the United States initiated a system whereby everyone would be treated that qualifies for social security benefits, after a waiting period of three years (Medicare Secondary Payer was slowly extended over the years to 36 months after diagnosis as it currently stands), regardless of age, providing the platform for the debate of a potential nationalized health care system that currently seems to be in a period of uncertainty. However, over three decades through significant consolidation, the treatment of dialysis via two large provider chains (one fully integrated) have directed patients to hemodialysis in the out-patient setting without considering the technological or clinical benefits of home hemodialysis or peritoneal dialysis [1,2]. This may have occurred as a result of medical training although the overall system is structured more on a corporate basis today with new nephrologists joining practices that are already contracted with a large chain through medical directorships. That said, patients seem to be directed towards outpatient hemo-dialysis over other options such as home hemo-dialysis and peritoneal dialysis. The result has created an economic and medical system that has shifted toward what is perceived as the lowest cost of care without a consideration for other treatment modalities that promote a better quality of life for the patient as well as greater monetary benefits for the United States Federal Government. From a pure economic standpoint, profitability for providers is in the out-patient clinics despite the initial investment of \$1-2 million to build out a clinic via fixed asset utilization and the variable cost of peritoneal dialysis as well as the supply costs needed for home hemodialysis. In other words, for these two treatment modalities, the margin, if any, is built purely into the treatment. Margins can be enhanced through high utilization of in-center facilities via leveraging the staff and fixed costs while using economies of scale to reduce variable costs such as dialyzers and lines combined with a favorable commercial patient base. With the focus on this modality, there are economic impacts based on decisions that increase the overall expenditures to the entire system that can easily be avoided or reduced.

\section{Overview}

There is no doubt that the treatment of renal failure is extremely complex and expensive requiring multiple medical approaches and disciplines each unique to the patient. However, from the perspective of treatment when it comes to renal failure, the standard approach has been to prescribe dialysis on an outpatient basis for those with kidney failure. As a mature industry, outpatient dialysis clinics have grown by $5.05 \%$ from 5,871 to 6,479 between 2010 and 2013 [3]. There is an obvious need for the neighborhood clinic, but two large dialysis providers, DaVita and Fresenius Medical Care (4,833 units), wouldn't invest in these multiple locations to similar companies like Burger King (7,500 units), unless the profitability was not evident and obviously continual for a reasonable financial payback and return [4]. As by comparison, the top ten providers, including DaVita and Fresenius, own 5,888 clinics with the bottom eight owning and controlling 1,055 clinics. Given movements and advancements in medical technology, the question is why the dominant number of patients that need dialysis remain with outpatient in-center and not home hemodialysis or peritoneal (CCPD or CAPD) dialysis. Trends in these modalities have been, at the very least stagnant with $9.7 \%$ of incidence patients being prescribed this treatment in 2016 and 2.8\% receiving a kidney transplant with prevalent hemo dialysis ESRD patients, without a transplant, 98\% using in-center hemo dialysis, and $2.0 \%$ using home hemo dialysis, according to the United States Renal Data System Annual Report. Companies such as Nx Stage, the leader in a home hemodialysis therapy machine, given the financial pressures that they are under, despite a purchase offer from Fresenius Medical Care, do not report their patient numbers 
in their annual reports combined with an accumulating loss from total perations of \$13.8 million in 2017 in light of a highly intuitive and revolutionary product [5]. From the Nx Stage perspective, this is a clearly almost an impossible market to participate in given that the two large service providers control almost $85 \%$ of the accessibility and the subsequent nephrologists that serve as medical directors of the top ten providers treating 383,464 patients of the 453,043 patients (non-transplant) in 2017. Numbers even for the top providers, which should frankly stand as a mark of where treatment modalities should be driven, are consistent with national averages for the entire market of dialysis caregivers, holding as only $2 \%$ of the combined providers' patients undergoing dialysis at approximately 6,200 patients in total [6]. Nephrologist groups, many of whom had sold their outpatient clinics to theses chains in the past twenty years, sign lucrative medical director deals going forward or either enter and agree to joint venture arraignments that incent the number of patients flowing into the clinic going forward and may influence physician groups to guide patients towards specific treatment modalities that benefit the entity and not the specific patient. Obviously, this sets up the stage for new nephrologists joining a practice that is already tied to a specific company for outpatient dialysis services for a medical director fee that is paid to the practice for services rendered. New nephrologists certainly do not have the opportunity to start their own clinic, as it once was envisioned under the mandate in 1972, so innovation and entrepreneurship has been shifted towards DaVita and Fresenius from the physician.

\section{The Economics of Dialysis}

End Stage Renal Disease has always been an expensive to treat. Prior to 1972, committees were established to decide what patients were best suited for treatment. The country has moved far past these committees where all patients have access to this life saving therapy. Removing hospitalizations and treatments outside the clinic, dialysis is extremely expensive despite improvements in technology and utilization of patients through a clinic. Skilled care combined with the infrastructure around a patient requires a set cost that will rise given inflation and other outside variables. The basic cost for treatment seems fairly strait forward given the location of the facility and the subsequent variations in expenditures adjusted for geographical regions. However, if the supply costs for in-center dialysis are substantially lower or at a reasonable comparison than peritoneal or home hemodialysis, than higher utilization of fixed costs, such as staff and rent, can yield lower overall costs than that of selling and providing peritoneal and home hemo supplies for patients despite obvious benefits in fixed costs as compared to an underutilized outpatient dialysis setting. The challenge with the analysis of the treatment of end stage renal disease from an economic standpoint has always been somewhat misdirected in that the focus has traditionally been on the hands on treatment and not the macro impact on the economy. As previously stated, we know that dialysis is both a necessary and an expensive treatment for survival. Economic or billable costs for treatment in the United States by the Medicare system (CMS) have topped $\$ 28$ billion or almost $\$ 91,000$ per patient for in-center hemodialysis or $\$ 35$ billion in total [7]. Medicare expenditures are consistently growing as a result of diabetes, hypertension and the aging population although net growth of mortality has slowed the last few years. However, this is only a fraction of the economic cost to the United States and the global economy. What needs to be considered is the total economic cost of patients undergoing dialysis by treatment modality. Simply, what is the quality of life for patients undergoing peritoneal dialysis, in-center hemodialysis, and daily home hemodialysis. If, given schedules and how a patient feels after experiencing one of these therapies, a patient is unable to stay in the workforce under the age of 65 , the patient becomes a negative impact on the economy. Patients that can stay in the workforce contribute via taxes and personal expenditures rather than transferring to the support system established by the government for disabilities.

\section{Profitability}

Part of the problem is incentives for the dialysis industry. True, the government was sold on transplantation as a savior for the system with costs only expected to be totaling $\$ 290$ million although the first year it was closer to a billion dollars only to rise significantly in the following years [3]. The entitlement was also supposed to be a model for what nationalized healthcare should look like. Expenditures and costs both exceeded expectations and didn't truly reflect what nationalized health care would look like given that this is chronic disease with little or no coverage for preventative care. What has transpired is a healthcare system with two large chain providers that dictate the modality of a patient towards in-center hemodialysis. In 1972, nephrologists were the owners and operators of dialysis clinics. Today, nephrologists join practices that are under contractual obligations to provide medical services to large chains. In some ways, this doesn't make sense. One would look at the expenditure of $\$ 1-\$ 2$ million for an outpatient clinic combined with staffing needs for patient treatment. Fresenius has eliminated the reuse of dialyzers via high volume production making it financially feasible to suspend this practice for patients. However, despite this advancement via cost savings in the in-center hemodialysis setting, assuming a higher utilization, the result is a focus on one treatment modality without considering PD or home hemodialysis. Certainly, it is easier for a nephrologist to prescribe an in-center modality given the risks associated with other treatment options such as peritoneal dialysis or home hemo therapies. The problem that surfaces from this option is one that drives patients towards a therapy that may reduce, patient quality of life and increased economic expenditures over the patient's lifespan. That said, not all patients are suitable for home dialysis therapies. Dialysis providers, from a financial perspective, embrace in-center hemo dialysis. This is clearly reflected in the recent treatment 
modalities whereby home hemo dialysis reflects such a minimal number of those treated, that virtually no analysis is conducted with regards to this modality [8]. With profitability driven towards in-center treatment, the economic benefits of other treatment modalities go un-noticed or merely provide the perspective of the "hands on provider" rather than the total economic expenditures to the respective health care system. This does not negate proper treatment recommendations by physicians given patient lifestyles and environments that may necessitate a different venue for dialysis. If, for example, a patient is suitable for home treatment modalities, even if conducted within an outpatient setting, while the variable costs via dialyzers and lines are twice the cost, patient lifestyle and economic output would remain higher given a reduced demand on government resources and contributions to said system through continued employment and contributions via economic output and taxes.

\section{Conclusion}

While the overall evidence reflects an environment that would favor more treatment modalities in the home setting when appropriate to reduce overall economic costs of dialysis, the composition of the industry being consolidated into two providers will likely prohibit any treatment modality shift for the patient population. The underlying assumption that markets would drive down costs via competition and consolidation in this case seems to be untrue and counterproductive to payers such as the Medicare system. To drive costs down for this important therapy may pose an economic conundrum. If supply costs such as dialyzers and lines are reduced, the result may re-enforce the drive towards in-center dialysis as the preferred modality further enhancing profitability for providers but at the same time not taking advantage of the overall economic picture. Action that needs to take place is somewhat obvious but also seems unlikely. Medicare (CMS) and medical schools both have to get in front of the disease with a prevention system keeping patients off of dialysis and embracing a system that promotes home therapies that reduces total economic exposure as well as increases individual outcomes and quality of life.

\section{References}

1. Incidence, Prevalence, Patient Characteristics, and Treatment Modalities, US Renal Data Systems, USA.

2. Christian Kronborg, Trine Kjær, Michael Bech (2010) Cost analysis of establishing dialysis facilities for the treatment of chronic renal failure in Greenland, International Journal of Circumpolar Health 69(5): 470-479.

3. United States Renal Data System (2018) USRDS annual data report: Epidemiology of kidney disease in the United States. National Institutes of Health, National Institute of Diabetes and Digestive and Kidney Diseases, Bethesda, USA.

4. (2019) Number of Burger King restaurants in the United States and Canada from 2011 to 2014.

5. (2017) NxStage Annual Report. United States Securities and Exchange Form 10K 2017.

6. (2017) The largest dialysis providers in 2017: More jump on integrated care bandwagon.

7. Shinkman Ron (2016) The Big Business of Dialysis Care. The New England Journal of Medicine.

8. Sullivan, John D (2019) The Current State of the Treatment of End Stage Renal Disease in 2019 in the United States. JJ Nephro Urol 5(1): 36.

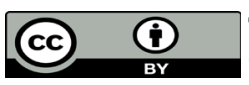

This work is licensed under Creative Commons Attribution 4.0 License

To Submit Your Article Click Here: Submit Article
DOI: $10.32474 / J U N S .2019 .01 .000119$

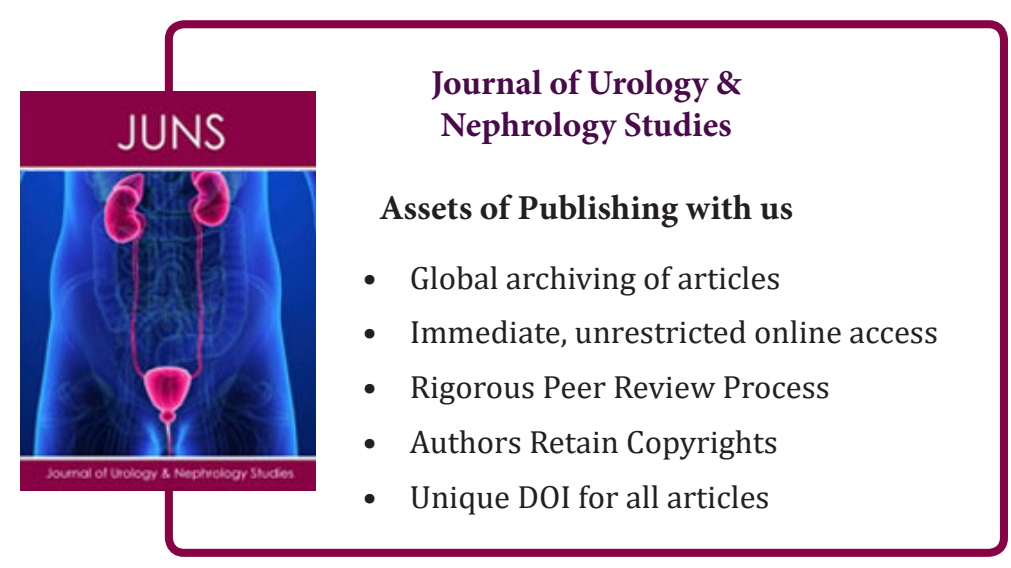

